\title{
Arcane Information, Solving Relations, and Church Censorship
}

\author{
Leonid A. Levin \\ Boston University \\ University of Heidelberg \\ Humboldt Foundation \\ http://www.cs.bu.edu/fac/lnd
}

\begin{abstract}
Church-Turing Thesis fails for problems that allow multiple answers: many easily solvable problems allow only non-recursive solutions. Its corrected version is: Physical and Mathematical Sequences have Little Common Information. This requires extending Kolmogorov's concept of mutual information to infinite strings. This is tricky; the talk will survey these and other related issues. Related Information can found at: http://arxiv.org/abs/cs.CC/0203029.
\end{abstract}

